\title{
Analysis of the Passive Damping Losses in LCL-filter Based Grid Converters
}

\author{
Rafael Peña-Alzola, Marco Liserre, Member, IEEE, Frede Blaabjerg, Fellow, IEEE, Rafael Sebastián, \\ Member, IEEE, Jörg Dannehl and Friedrich Wilhelm Fuchs, Senior Member, IEEE
}

\begin{abstract}
Passive damping is the most adopted method to guarantee the stability of $L C L$-filter based grid-converters. The method is simple and, if the switching and sampling frequencies are sufficiently high, the damping losses are negligible. This letter proposes the tuning of different passive damping methods and an analytical estimation of the damping losses allowing the choice of the minimum resistor value resulting in a stable current control and not compromising the $L C L$-filter effectiveness. Stability, including variations in the grid inductance, is studied through root locus analysis in the $z$-plane. The analysis is validated both with simulation and with experiments.
\end{abstract}

Index Terms - Passive damping, $L C L$-filter, stability, voltagesource converter.

\section{INTRODUCTION}

$\mathrm{P}$ ASSIVE damping is the most adopted method to guarantee the stability of the $L C L$-filter based grid-converters. Neither new sensors nor changes in the control software for the $L$-filter case are necessary but there are additional encumbrances and losses that could claim for forced cooling [1]. Active damping modifies the control to obtain stability without using dissipative elements. The damping losses are avoided at the price of increased complexity in the control design and/or implementation [2]-[3], moreover sometimes new sensors are added [4]-[5]. This letter reviews the passive damping methods [6]-[7] and proposes an analytical estimation of the losses to select the best configuration resulting in stability without compromising the $L C L$-filter effectiveness. Stability analysis, including for grid inductance variations, uses root locus in the $z$-plane.

Manuscript received April 27, 2012.

R. Peña-Alzola is with the Department of Energy Technology, Aalborg University, DK-9220 Aalborg East, Denmark (e-mail: rpa@et.aau.dk).

M. Liserre is with the Department of Energy Technology, Aalborg University, DK-9220 Aalborg East, Denmark (e-mail: mli@et.aau.dk).

F. Blaabjerg is with the Department of Energy Technology, Aalborg University, DK-9220 Aalborg East, Denmark (e-mail: fbl@et.aau.dk).

R. Sebastián is with the Department of Electrical, Electronic and Control Engineering, National University for Distance Learning, UNED, 28040Madrid, Spain (e-mail: rsebastian@ieec.uned.es).

J. Dannehl is with Danfoss Solar Inverters A/S, DK-6400 Sønderborg, Denmark (e-mail: dannehl@danfoss.com).

F. W. Fuchs is with the Institute for Power Electronics and Electrical Drives, Christian-Albrechts-University of Kiel, 24143 Kiel, Germany (e-mail: fwf@tf.uni-kiel.de).

\section{Passive Damping Design}

\section{A. Simple resistor case}

Fig. 1 shows a three phase $L C L$-filter based converter with passive damping for the simple resistor case. The transfer function $G_{p d}(s)$, related to the converter voltage $v$ and the converter current $i$, is [8]:

$G_{p d}(s)=\frac{i(s)}{v(s)}=\frac{1}{L s} \frac{s^{2}+2 \zeta^{\prime} z_{L C} s+z_{L C}^{2}}{s^{2}+2 \zeta \omega_{r e s} s+\omega_{r e s}^{2}}$

with $z_{L C}^{2}=\left(L_{g} C_{f}\right)^{-1}, \quad \omega_{\text {res }}^{2}=\left(2 \pi f_{\text {res }}\right)^{2}=\left(1+L_{g} / L\right) z_{L C}{ }^{2} \quad$ is the resonance frequency, $L_{g}$ the grid inductance, $L$ the converter inductance and $C_{f}$ the filter capacitance. The damping factors are calculated as:

$\zeta=\frac{C_{f} \omega_{r e s}}{2} R_{d}$

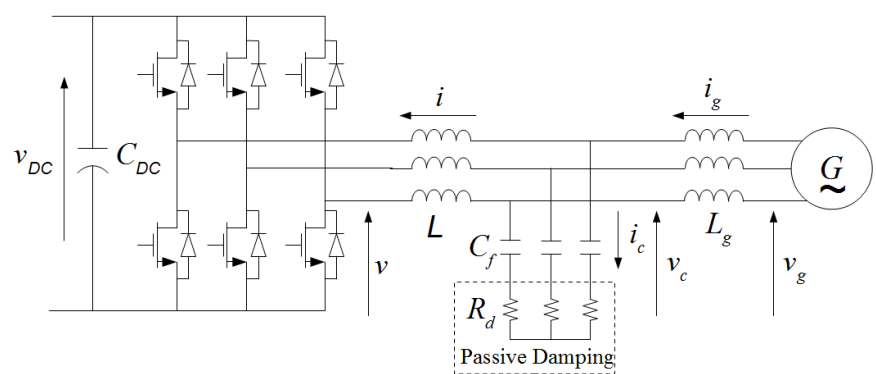

Fig. 1. $L C L$-filter based three phase converter using passive damping (simple resistor case).

and $\zeta^{\prime}=R_{d} C_{f} z_{L C} / 2$ with $R_{d}$ the damping resistor value. From (2) $R_{d}$ has the order of magnitude of the $L C L$-filter capacitor impedance at $\omega=\omega_{\text {res }}$. In the lower power range the switching frequency $\omega_{s w}=2 \pi f_{s w}$ is selected larger than $\omega_{\text {res }}$ [9]. The transfer function related to the grid current $i_{g}$ and $v$ has a zero at $z=-1 / R_{d} C_{f}$ which decreases the attenuation for $\omega>>\omega_{\text {res }}$ from $60 \mathrm{~dB} /$ decade, see Fig. 2 for the case with no damping, down to $40 \mathrm{~dB} /$ decade, see Fig. 2 for the simple resistor case. Thus, $R_{d}$ should be lower, or at least not much higher, than:

$$
R_{d s w}=\frac{1}{C_{f} 2 \pi f f_{s w}}
$$


where $R_{d s w}$ is the impedance of the filter capacitor at $f_{s w}$ so $\omega_{s w}<<1 / R_{d} C_{f}$. The current control uses a rotating $d q$-frame synchronous to the grid frequency. The current control uses a rotating dq-frame synchronous to the grid frequency. When considering the converter current $i$ as feedback with a simple digital PI controller, the open loop transfer function results in:

$$
\left|G_{o l}(s)\right|_{s=j \omega_{r e s}}=\left|G_{P I}(s) G_{\text {delays }}(s) G_{p d}(s)\right|_{s=j \omega_{r e s}}=1(0 \mathrm{~dB})
$$

where $G_{\text {delays }}(s)$ models the computational delay (one sampling period $T_{s}=1 / f_{s}$ ) and the PWM delay (half sampling period) and $G_{P I}(s)$ is the PI controller.

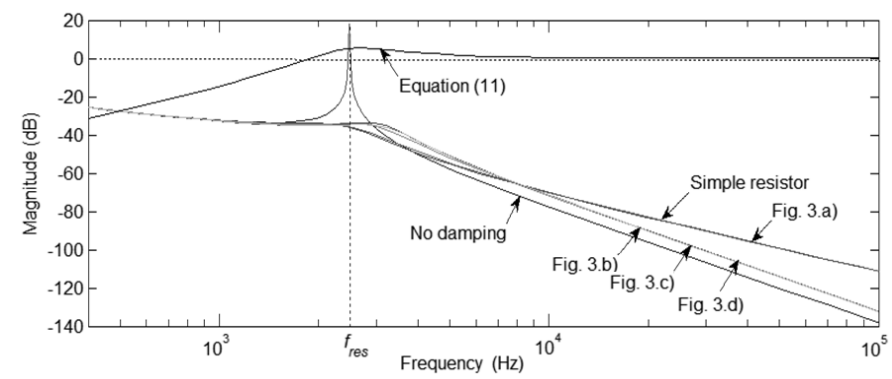

Fig. 2. Frequency response of the grid current $i_{g}$ versus the converter voltage $v$ (case with no damping, simple resistor case and configurations shown in Fig. 3 ) and the ratio between $i_{c}$ and $\hat{\imath}_{\mathrm{c}}$ expressed in (11).

At low frequency the capacitor branch can be neglected and the $L C L$-filter behaves as an $L$-filter with the inductance value as $L_{T}=L+L_{g}$ the sum of the grid and converter inductances. This control dynamics remains unchanged with the introduction of passive damping, see [1] for details. Hence, the PI controller is tuned using the technical optimum criterion with integration time $T_{i}=L_{T} / R_{T}$ and proportional gain $k_{p}=L_{T} /\left(3 T_{s}\right)$ [8] for $4 \%$ overshoot (damping factor $\zeta=0.707$ ) where $R_{T}=R+R_{g}$ is the sum of the grid and converter inductor resistances $R$ and $R_{g}$ respectively. As the phase-shift of $-180^{\circ}$ in $G_{o l}(s)$ is near $\omega=\omega_{\text {res }}$ [8], [10], the minimum damping resistor $R_{d m i n}$ for a stable system must comply (4) in order to achieve a positive gain margin in the control. To obtain a simple estimation for $R_{d m i n}$ the effect of the delays is neglected as $\left|G_{\text {delays }}\left(j \omega_{\text {res }}\right)\right| \approx 1$ for $\omega_{\text {res }}<2 \pi f_{s}$ and the numerator of (1) is approximated to $\left|s^{2}+2 \zeta^{\prime} z_{L C} s+z_{L C}{ }^{2}\right| \approx\left|s^{2}+z_{L C}\right|$ as $\omega_{\text {res }}>z_{L C}$. After neglecting the smallest terms, it results in:

$R_{d \min }=\frac{1}{3} f_{s} \frac{L_{g}^{2}}{\left(L+L_{g}\right)}=\frac{1}{6 \pi} \frac{L_{g}}{L} \frac{f_{s}}{f_{\text {res }}}\left(\frac{1}{C_{f} \omega_{\text {res }}}\right)$

From the assumed simplifications (5) will be accurate in the case of $f_{s}>>f_{\text {res }}$ and $L_{g} / L>>1$. For $f_{s} \approx 2 f_{\text {res }}$ and $L_{g} / L<2, R_{\text {dmin }}$ will be approximately $20 \%$ of the capacitor impedance value at $\omega=\omega_{\text {res }}$ which is coherent with the recommended value for the damping resistor [1] of about one-third of that value.

\section{B. Complex passive damping methods}

The damping losses $P_{d}$ can be reduced by using additional passive elements in the capacitor branch, see Fig. 3. In Fig. 3a at the fundamental frequency $\omega=\omega_{f}$ the inductance $L_{d}$ in parallel with the resistor must provide a low impedance, $L_{d} \omega_{f}<<R_{d}$ [7], so the fundamental losses $P_{d f}$ are nullified. At $\omega=\omega_{\text {res }}$ the resistor must have the dominant current flow path, $R_{d}<<L_{d} \omega_{r e s}$, for proper damping. The frequency response for Fig. 3a, and so the grid current THD, is very similar to that of the simple resistor case, see Fig. 2. In order to attain a proper trade-off the impedance ratio of the resistor $R_{d}$ and the inductor $L_{d}$ at $\omega=\omega_{f}$ and $\omega=\omega_{\text {res }}$ are made equal as:

$\frac{R_{d}}{L_{d} \omega_{f}}=\frac{L_{d} \omega_{r e s}}{R_{d}}$

Fig. $3 \mathrm{~b}$ adds a parallel capacitor $C_{d}$ to Fig. 3a for reducing the harmonic damping losses $P_{d H}$ [9] by short-circuiting the current harmonics around $\omega=\omega_{s w}$ so $1 /\left(C_{d} \omega_{s w}\right)<<R_{d}$ [7]. At $\omega=\omega_{\text {res }}$ the resistor must be the dominant current flow path, $R_{d}<<1 /\left(C_{d} \omega_{r e s}\right)$, for a proper damping. As previously discussed, $C_{d}$ can be:

$$
\frac{1 /\left(C_{d} \omega_{r e s}\right)}{R_{d}}=\frac{R_{d}}{1 /\left(C_{d} \omega_{s w}\right)}
$$

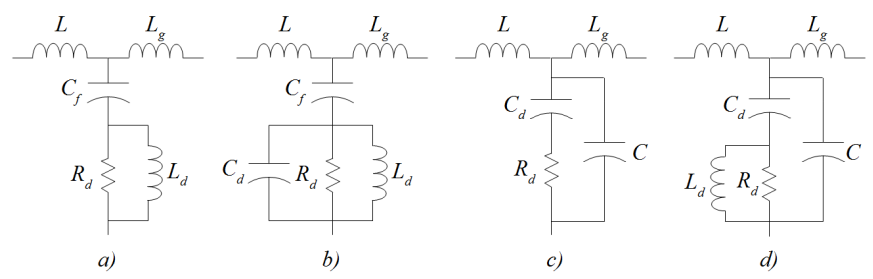

Fig. 3. Different configurations for passive damping.

\section{Estimation OF PASSIVE DAMPING Losses}

\section{A. Simple resistor case}

As $R_{d}<<Z_{c f}$, with $Z_{c f}$ the impedance of the filter capacitor at $\omega=\omega_{f}, R_{d}$ can be neglected to calculate the capacitor fundamental current $I_{c f}$. Thus, $P_{d f}$ for unity power factor can be estimated as:

$P_{d f} \approx 3 I_{c f}^{2} R_{d}=3\left(\frac{V_{c f}}{Z_{c f}}\right)^{2} R_{d}=3 \frac{\left[\left(V_{\text {nom }} / \sqrt{3}\right)^{2}+\left(\omega_{f} L_{g} I_{\text {nom }}\right)^{2}\right]}{1 /\left(\omega_{f} C_{f}\right)^{2}} R_{d}$

with $V_{\text {nom }}$ and $I_{\text {nom }}$ as the rated voltage and current respectively and $V_{c f}$ is the fundamental capacitor voltage. As usually $C_{f}<0.05 \mathrm{pu}$ in order not to decrease the power factor [1] too much, $I_{c f}$ will be small, and thereby $P_{d f}$ in the low power range. For $\omega \gg>\omega_{\text {res }}$ the converter harmonic currents find a negligible impedance path in the capacitor branch. Hence, the converter connected to the converter inductance $L$ can be used as an equivalent circuit for estimating the harmonic capacitor current $i_{c}$. The rms value of the inductor harmonic current for the usual space vector modulation was derived in [11], so the rms value of the estimated harmonic capacitor current $\hat{l}_{\mathrm{c}}$ is: 
$\hat{I}_{c H \text { RMS }}^{\text {lower }}=\frac{1}{2 \sqrt{3}} \frac{1}{\sqrt{48}} \frac{V_{D C}}{f_{s w} L} \sqrt{\left[\frac{3}{2} m^{2}-\frac{4 \sqrt{3}}{\pi} m^{3}+\frac{9}{8}\left(\frac{3}{2}-\frac{9}{8} \frac{\sqrt{3}}{\pi}\right) m^{4}\right]}$ (9)

where $m$ is the modulation index calculated by neglecting the $L C L$-filter capacitor branch at $\omega=\omega_{f}$ as:

$$
m=\frac{2 \sqrt{2}}{V_{D C}} \sqrt{\left(\frac{V_{n o m}}{\sqrt{3}}\right)^{2}+\left[\omega_{f}\left(L+L_{g}\right) I_{n o m}\right]^{2}}
$$

The ratio between $i_{c}$ and $\hat{l}_{\mathrm{c}}$ for the different harmonic frequencies $n \omega_{f}$, with the $n^{\text {th }}$ harmonic order, is:

$\left|\frac{i_{c}(s)}{\hat{i}_{c}(s)}\right|_{s=j n \omega_{f}}=\left|\frac{G_{c}(s)}{\hat{G}_{c}(s)}\right|_{s=j n \omega_{f}}=\left|\frac{\frac{s}{L\left(s^{2}+2 \zeta \omega_{\text {res }} s+\omega_{\text {res }}^{2}\right)}}{\frac{1}{L s}}\right|=\left|\frac{I_{c}\left(n \omega_{f}\right)}{\hat{I}_{c}^{\text {lower }}\left(n \omega_{f}\right)}\right|$

where $\hat{G}_{c}(s)$ is the transfer function related to $\hat{\imath}_{\mathrm{c}}$ and $v$ as well as $G_{c}(\mathrm{~s})$ is that related to $i_{c}$ and $v$. It can be seen in Fig. 2 that the frequency response of $(11)$ is more than unity $(>0 \mathrm{~dB})$ for $\omega>>\omega_{\text {res }}$ and approaches to unity $(0 \mathrm{~dB})$. Then (9) is a lower bound of the $L C L$-filter capacitor rms harmonic current $I_{c H R M S}$ for $\omega_{s w}>\omega_{\text {res }}$. Thus, a lower approximation $P_{d H}^{\text {lower }}$ for the harmonic damping losses $P_{d H}$ is:

$$
P_{d H}^{\text {lower }}=3\left(I_{c H ~ R M S}^{\text {lower }}\right)^{2} R_{d}
$$

Converter voltage harmonics, and so the current harmonics, appear as sidebands centered on the switching frequency and its multiples for linear modulation [11]. The current harmonic of order $m_{f}-6$ is negligible for a large frequency modulation ratio $m_{f}=\omega_{f} / \omega_{s w}$. Assuming $\left(m_{f}-6\right) \omega_{f}>\omega_{\text {res }}$, an upper bound of $I_{\text {CHRMS }}$ results:

$$
\hat{I}_{c H R M S}^{\text {upper }}=\hat{I}_{c H R M S}^{\text {lower }}\left|\frac{G_{c}(s)}{\hat{G}_{c}(s)}\right|_{s=j\left(m_{f}-6\right) \omega_{f}}=\hat{I}_{c H R M S}^{\text {lower }}\left|\frac{I_{c}\left(\left(m_{f}-6\right) \omega_{f}\right)}{\mid \hat{I}_{c}^{\text {lower }}\left(\left(m_{f}-6\right) \omega_{f}\right)}\right|
$$

Thereby, an upper approximation $P_{d H}^{\text {upper }}$ for the harmonic damping losses $P_{d H}$ results in:

$P_{d H}^{\text {upper }}=3\left(I_{c H R M S}^{\text {upper }}\right)^{2} R_{d}$

For elevated $f_{s w},(12)$ and (14) are very close to each other. The average of these values can be used to estimate $P_{d H}$.

Although $R_{d \min }$ for stability is proportional to $f_{s}$ in (5), $P_{d H}$ is inversely proportional to $f_{s w}$ in (12). However, $f_{s w}$ should not just be increased, since the switching losses are proportional to $f_{s w}$. Hence, the passive damping is an attractive approach versus active damping when elevated switching frequency is possible since the resulting damping losses are much reduced. For the same $f_{s w}$, using double PWM update mode $\left(f_{s}=2 f_{s w}\right)$, it doubles the bandwidth [10] but doubles also $R_{\text {dmin }}$ in (5) thereby increasing the losses. As usually $\left(L+L_{g}\right)<0.1 \mathrm{pu}[1], V_{c f}$ will be close to $V_{\text {nom }}$ in (8) and $m$ will vary a little with the load in (10). Thus, $P_{d}$ varies slightly with the load unlike the switching losses which are proportional to the load current.

\section{B. Complex passive damping methods}

In Fig. $3 \mathrm{~b}$ the current through the whole capacitor branch can be estimated with (9) and (13) with $G_{c}(s)$ calculated for the present configuration. As most important harmonics are around $\omega=\omega_{s w}$, the current through the damping resistor can be estimated by the ratio of impedances at $\omega=\omega_{s w}$. Therefore:

$P_{d} \approx P_{d H}\left|\frac{Z_{d}}{R_{d}}\right|^{2}$

where $Z_{d}=Z_{C_{d}}\left\|Z_{R_{d}}\right\| Z_{L_{d}} \approx Z_{C_{d}} \| Z_{R_{d}}$ are impedances for $\omega=\omega_{s w}$. Equation (15) is an upper approximation since higher harmonics will have lower ratios $\left|Z_{d} / R_{d}\right|$. The frequency response for Fig. $3 b$ does not degrade for $\omega>>\omega_{\text {res }}$ as much as in the simple resistor case and so the grid current THD improves, see Fig. 2.

In Fig. 3c most of the current harmonics bypasses the damping resistor branch by circulating through the parallel capacitor $C$ [12]. $P_{d}$ is reduced although the resistor value for the proper damping is higher than that of the simple resistor case. Reference [12] proposes equal capacitances in both branches $C_{d}=C=C_{f} / 2$. Again $R_{d}$ can be neglected in order to calculate $I_{c f}$ since $R_{d} \ll<1 /\left(C_{d} \omega_{f}\right)$ and, with two parallel branches for $C_{d}$ and $C$, the resulting current through $R_{d}$ is halved so $P_{d f}$ for Fig. $3 \mathrm{c}$ is a quarter of (8). To calculate $P_{d H}$ for Fig. $3 c$ it must be proceeded as in (15) by considering the main harmonics around $\omega=\omega_{s w}$. Hence, $P_{d}$ results in:

$P_{d} \approx \frac{P_{d f}}{4}+P_{d H}\left|\frac{Z_{C T}}{Z_{d}}\right|^{2}$

where $Z_{d}=R_{d}+Z_{C d}$ and $Z_{C T}=Z_{C} \| Z_{d}$ are also the impedances for $\omega=\omega_{s w}$. As previously discussed, (16) is also an upper approximation. In Fig. 3d [6] the inductor $L_{d}$ is located in parallel to the damping resistor shown in Fig. $3 \mathrm{c}$ once more to annul the fundamental losses $P_{d f}$. The frequency responses for the circuits in Fig. 3c and Fig. 3d are very similar to that for the circuit in Fig. 3b, see Fig. 2.

Finally, the insertion of an additional computational delay in the control loop plays a positive role since it results in a more stable system [8]. Therefore, for the same damping in the closed loop poles, the necessary value of $R_{d}$ in the simple resistor case can be halved, and so also the power losses. However, this is at the expense of reducing the bandwidth to $60 \%$, all the details can be found in [8]. 


\section{SimULATION AND EXPERIMENTAL RESULTS}

The data of the $L C L$-filter based grid converter are: $L_{g}=5$ $\mathrm{mH}, L=3 \mathrm{mH}, C_{f}=2.2 \mu \mathrm{F}, P_{\text {nom }}=4.1 \mathrm{~kW}$ and $V_{\text {nom }}=380 \mathrm{~V}$. All details about the experimental setup along with relevant waveforms can be found in [1]. Table I shows that the estimation $R_{d \min }(5)$ of the minimum damping resistor for stability is conservative and accurate only for elevated $f_{s w}$ as expected from the simplifications used. Table II and Table III show that the estimated losses are very close to those obtained by simulation (Matlab/Simulink). This is because the simulation models of the power devices and the passive elements are ideal. As expected from the assumptions used, the accuracy increases for increasing $f_{s w}$. Table III also shows the grid current THD obtained by simulation.

TABLE I. MINIMUM DAMPING RESISTOR FOR STABILITY.

\begin{tabular}{lllll}
\hline$f_{s w}$ & $6 \mathrm{kHz}$ & $7 \mathrm{kHz}$ & $8 \mathrm{kHz}$ & $9 \mathrm{kHz}$ \\
\hline Calculated & $2.6 \Omega$ & $5.1 \Omega$ & $7.2 \Omega$ & $8.9 \Omega$ \\
$R_{d \min }(5)$ & $6.3 \Omega$ & $7.3 \Omega$ & $8.3 \Omega$ & $9.4 \Omega$ \\
\hline
\end{tabular}

TABLE II. DAMPING LOSSES OBTAINED BY SIMULATION AND ESTIMATION FOR THE SIMPLE RESISTOR CASE $\left(R_{D}=10 \Omega\right)$.

\begin{tabular}{ccccc}
\hline$f_{s w}$ & $5 \mathrm{kHz}$ & $6 \mathrm{kHz}$ & $7 \mathrm{kHz}$ & $8 \mathrm{kHz}$ \\
\hline Simulation(W) & 41.5 & 25.8 & 18.0 & 13.4 \\
Estimation(W) & 41.1 & 25.4 & 17.7 & 13.2 \\
\hline
\end{tabular}

TABLE III. DAMPING LOSSES, SIMULATION AND ESTIMATION, GRID CURRENT THD, SIMULATION, AND GRID INDUCTANCE VARIATION RESULTING IN INSTABILITY FOR THE DIFFERENT CONFIGURATIONS $\left(f_{s w}=8 \mathrm{kHz}\right)$.

\begin{tabular}{cccccccc}
\hline & Simple & Double & Fig. & Fig. & Fig. & Fig. & Add. \\
& Resistor & Update & $3 \mathrm{a}$ & $3 \mathrm{~b}$ & $3 \mathrm{c}$ & $3 \mathrm{~d}$ & Delay \\
\hline Simulation(W) & 21.1 & 33.2 & 19.8 & 4.1 & 4.9 & 3.7 & 9.4 \\
\hline Estimation $(\mathrm{W})$ & 20.9 & 33.0 & 19.8 & 5.3 & 6.2 & 4.8 & 9.3 \\
\hline$i_{g} \mathrm{THD}(\%)$ & 1.5 & 1.0 & 1.5 & 0.8 & 0.8 & 0.8 & 0.8 \\
\hline$L_{\text {greal }} / L_{g}$ & 7.5 & - & 6.6 & 3.6 & 3.7 & 6.7 & 5.8 \\
\hline
\end{tabular}

Fig. 4 shows the $z$-plane root locus for increasing $R_{d}$. Stability is attained for $R_{d m i n}=8.3 \Omega(5)$, the threshold is $R_{d s w}=9$ $\Omega$ (3) and a proper damping ( $\zeta_{c} \approx 0.1[8]$ ) needs $R_{d}=16 \Omega$. Double update mode doubles the bandwidth up to $848 \mathrm{~Hz}$ but it needs $R_{d}=26 \Omega$ and thereby increasing the damping losses. Although $R_{d}>>R_{d w}$ this elevated bandwidth lowers the grid current THD. The reduction in $P_{d}$ for Fig. $3 \mathrm{a}$ with $L_{d}=7.2 \mathrm{mH}$ (6) is small since $P_{d f}$ was only $1.1 \mathrm{~W} . P_{d}$ and the grid current THD are substantially reduced for Fig. 3b with $C_{d}=2.2 \mu \mathrm{F}$ (7). Fig. 3c needs $R_{d}=80 \Omega$, notwithstanding, $P_{d}$ and the current grid THD are much reduced. As expected (15) and (16) used for Figs. 3b, 3c and 3d result in upper approximations for $P_{d}$. Fig. $3 \mathrm{~d}$ with $L_{d}=36 \mathrm{mH}$ (6) produces a modest improvement since $P_{d f}$ was only $1.3 \mathrm{~W}$. Inserting an additional delay requires $R_{d}=7 \Omega$ with a reduced bandwidth, $255 \mathrm{~Hz}$, but an improved grid current THD as now $R_{d}<R_{d s w}$.

Fig. 5 shows the root locus in the $z$-plane by varying the real grid inductance $L_{\text {greal }}$ for the simple resistor case. Stability is assured for decreasing $L_{\text {greal }}$ with $L_{\text {greal }}=0$, the $L$-filter case, inherently stable. Increasing $L_{\text {greal }}$ increases the damping of the dominant poles which resuls in lower overshoots and decreases the damping of the high frequency poles (2) until the system becomes unstable. However, this requires $L_{\text {greal }} / L_{g}>>1$ to reduce $\omega_{\text {res }}$ since the $L C L$-filter is designed with $L_{g} / L>1$ [1].
The analysis can be generalized for all the configurations except the double update mode that is stable for all $L_{\text {greal }}$, see Table III last row. The benefits of the circuits in Figs. $3 \mathrm{~b}$ and Fig. $3 \mathrm{c}$ are at the price of reduced robustness. Finally, an additional control delay reduces the stability as it was expected.

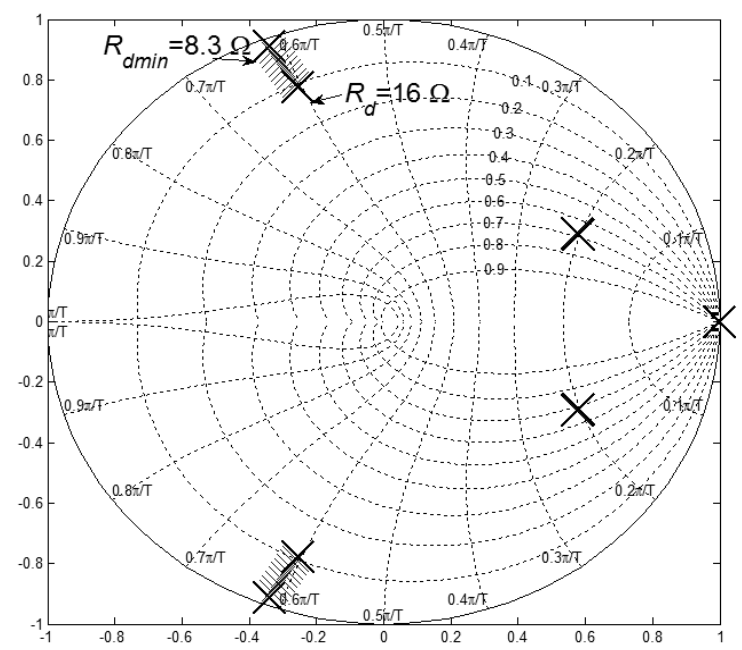

Fig. 4. Closed loop poles in the $z$-plane of the converter current control $\left(f_{s}=f_{s w}=8 \mathrm{kHz}\right)$ by varying the damping resistor value between $R_{d m i n}=8.3 \Omega$ and $R_{d}=16 \Omega$.

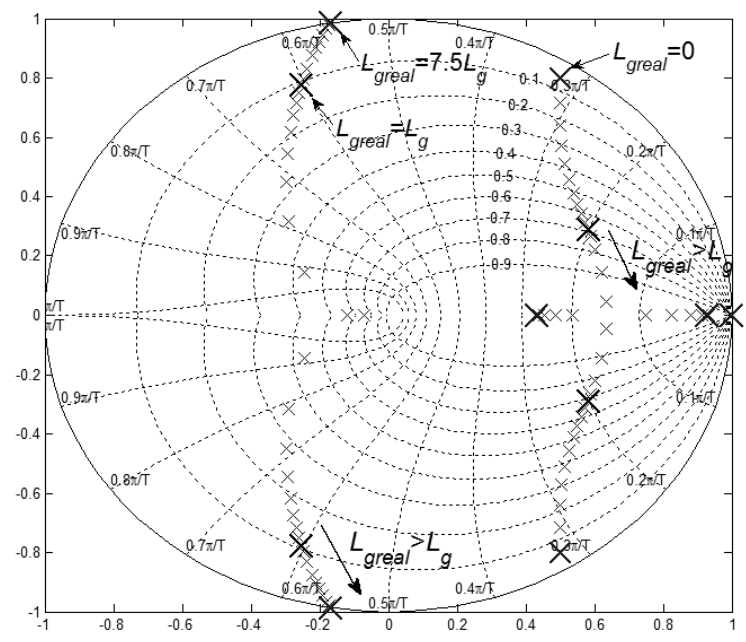

Fig. 5. Closed loop poles in the $z$-plane of the converter current control ( $f_{s}=f_{s w}=8 \mathrm{kHz}$ ) for $R_{d}=16 \Omega$ by varying the grid inductance between $L_{\text {greal }}=0$ and $L_{\text {greal }}=7.5 L_{g}$.

Finally, Table IV shows the values for $P_{d}$ by experiments in the simple resistor case to assess the validity of (12) and (14), the only equations not based on simple impedance relations for different frequencies. The values for $P_{d}$ indicated in Table IV are lower than those in Table II. $P_{d}$ estimated by experiments is close to the lower estimation given in equations (8) and (12) and even inferior for the lower $f_{s w}$ where the damping losses are higher. This is because the non-linearities of the passive elements (saturation, core losses, etc.), which further limit the harmonics, were not considered. Therefore, the proposed analysis can be safely used. 
TABLE IV. DAMPING LOSSES OBTAINED BY EXPERIMENTS FOR THE SIMPLE RESISTOR CASE $\left(\mathrm{R}_{\mathrm{D}}=10 \Omega\right)$.

\begin{tabular}{ccccc}
\hline$f_{s w}$ & $5 \mathrm{kHz}$ & $6 \mathrm{kHz}$ & $7 \mathrm{kHz}$ & $8 \mathrm{kHz}$ \\
\hline Lower estimation $(8)+(12)$ & 29.5 & 20.7 & 15.4 & 12.0 \\
Experimental(W) & 32 & 20 & 13 & 10 \\
\hline
\end{tabular}

\section{CONCLUSIONS}

This letter has focused on passive damping of $L C L$-filter based grid converters. The damping resistor value must result in stability without compromising the filter effectiveness. The provided analysis allows selecting this value and estimating the losses. The analysis is expanded to more complex methods that reduce the fundamental and/or harmonic components of the damping losses by using additional passive elements. The analysis in the z-plane shows the different robustness of each method when increasing the real grid inductance until the system becomes unstable.

\section{REFERENCES}

[1] M. Liserre, F. Blaabjerg and S. Hansen: "Design and control of an $L C L$ filter based three-phase active rectifier," in IEEE Trans. on Ind. Applicat., vol.41, no.5, pp. 1281- 1291, Sept.-Oct. 2005.

[2] Y.A.-R.I. Mohamed, M. A-Rahman and R. Seethapathy: "Robust linevoltage sensorless control and synchronization of LCL-filtered distributed generation inverters for high power quality grid connection," IEEE Trans. on Power Electron., vol.27, no.1, pp.87-98, Jan. 2012.

[3] Y. Tang, P.C. Loh, P. Wang, F.H. Choo and F. Gao: "Exploring Inherent Damping Characteristic of LCL-Filters for Three-Phase Grid-Connected Voltage Source Inverters," IEEE Trans. on Power Electron., vol.27, no.3, pp.1433-1443, March 2012.

[4] M. Xue, Y. Zhang, Y. Kang, Y. Yi, S. Li and F. Liu: "Full Feedforward of Grid Voltage for Discrete State Feedback Controlled Grid-Connected Inverter With LCL Filter," IEEE Trans. on Power Electron., vol.27, no.10, pp.4234-4247, Oct. 2012.

[5] J. He and Y.W. Li: "Generalized Closed-Loop Control Schemes with Embedded Virtual Impedances for Voltage Source Converters with LC or LCL Filters," IEEE Trans. on Power Electron., vol.27, no.4, pp.1850-1861, April 2012

[6] K.H. Ahmed, S.J. Finney and B.W. Williams: "Passive Filter Design for Three-Phase Inverter Interfacing in Distributed Generation," in Compatibility in Power Electron., CPE '07, pp. 1-9, 2007.

[7] T.C.Y. Wang, Zhihong Ye, Gautam Sinha and Xiaoming Yuan: "Output filter design for a grid-interconnected three-phase inverter," in IEEE 34th Annu. Power Electron. Specialist Conf., PESC '03, vol. 2, pp. 779784, 15-19 June 2003.

[8] M. Liserre, A. Dell'Aquila and F. Blaabjerg: "Stability improvements of an $L C L$-filter based three-phase active rectifier," in 2002 IEEE 33rd Annu. Power Electron. Specialists Conf., PESC'02. Vol. 3, pp. 11951201, 2002.

[9] A.A. Rockhill, M. Liserre, R. Teodorescu and P. Rodriguez: "Grid-Filter Design for a Multimegawatt Medium-Voltage Voltage-Source Inverter," in IEEE Trans. on Ind. Electron., vol.58, no.4, pp.1205-1217, April 2011.

[10] V. Blasko and V. Kaura: "A novel control to actively damp resonance in input LC filter of a three-phase voltage source converter," in IEEE Trans. on Ind. Applicat., vol.33, no.2, pp.542-550, Mar 1997.

[11] D.G. Holmes and T.A. Lipo: Pulse width modulation for power converters: principles and practice, Chapter 6. Pages: 291-294, WileyIEEE Press, October 2003. ISBN: 978-0-471-20814-3.

[12] P. Channegowda and V. John: "Filter optimization for grid interactive voltage source inverters," in IEEE Trans. on Ind. Electron., vol. 57, no. 12, pp. 4106-4114, Dec. 2010. 